\title{
A possible binary nuclei in Mrk 622
}

\section{J.M. Rodríguez Espinosa*}

Instituto de Astrofísica de Canarias

Universidad de La Laguna

E-mail: jrediac.es

E. Benitez

Instituto de Astrononomía

UNAM

México

E-mail: erikadastro.unam.mx

\section{Cruz-González}

Instituto de Astrononomía

UNAM

México

E-mail: irene@astro.unam.mx

O. González Martín

CRYA

Morelia, Michoacan

México

E-mail: o.gonzalez@crya.unam.mx

\section{A. Negrete}

Instituto de Astrononomía

UNAM

México

D. Ruschel-Dutra

Dept. de Astronomia

Universidade Federal do Rio Grande do Sul

Brasil

\section{Gutierrez}

Instituto de Astrononomía

UNAM

México

\section{E. Jimenez-Bailon}

Instituto de Astrononomía

UNAM

México 
We have performed optical spectroscopy of Mrk 622 finding that it shows tripled peaked features. These features are separated in velocity showing two components that are blue-shifted and reshifted with respect to the systemic velocity, and a third component that is right at the systemic velocity. Besides, when analysing their spectra we find that the ratio of the [OIII] to $\mathrm{H}_{\beta}$ and [NII] to $\mathrm{H}_{\alpha}$ lay in the locus of AGN for the blue and red-shifted components, while the systemic velocity component lays at the starburst/HII locus. This is interpreted as having a binary AGN that is orbiting a central Starburst.

Frontier Research in Astrophysics III Workshop

28 May -1 June, 2018

Palermo, Italy

* Speaker. 


\section{Introduction}

The current paradigm of galaxy formation states the galaxies grow through mergers ([1], [16],[23], [20]). Since bulge dominated galaxies tend to have nuclear super massive Black Holes (SMBH), there should be many instances of binary AGN. However so far the evidence for binary AGN has been scant $([7,8])$. The Sloan Digital Sky Survey (SDSS) has found many AGN that show double peaked emission lines in their spectra ([5], [21], [9], [6], [2]), that could be interpreted as coming from two merging AGN ([25], [8]). However, the interpretation is not unique, as double peaked emission lines could be produced by rotating disks as well as by winds or outflows ([10], [22], [7], [17]). Here we show that Mrk622 is a triple peaked galaxy, with both blue and red shifted narrow emission lines, as well as a narrower emission line at the systemic velocity. The blue and red shifted lines are in the locus of AGN in a Baldwin, Phillips \& Terlevich (BPT) diagram ([13], [12]). The central line falls instead in the locus of star forming galaxies. All of this is occurring within $76 \mathrm{pc}$. Based on the above, we conclude that Mrk 622 is a binary AGN where the two nuclei are separated by a star forming region. This region precludes the possibilities of having either a rotating disk, winds, or outflows, as these would heat up the gas and increase its turbulence, thus preventing star formation. This resembles the case of the discovery long ago of "Spectroscopic Variables". Here instead we find the presence of an unresolved double nuclei through spectroscopy.

\section{Markarian 622}

Mrk 622, also known as UGC 4229, has a $\mathrm{z}=0.023$ and is classified as an S0-pec Seyfert 2 (Sy2) galaxy according to NED ${ }^{1}$. Among the first spectroscopic studies of this AGN, stands out the comment that this object shows emission lines with multiple components ([18]). In a recent study done with Herschel PACs ([25]), these authors found that this object is a composite AGN plus starburst (SB) galaxy. Furthermore, the Spitzer/IRS spectrum shows that Mrk622 has a Sy2 mid-IR spectrum ([25]) with strong PAH emission features.

We observed Mrk622 to study the origin of the double peak narrow emission lines ( [21]). We obtained new high S/N optical spectroscopy with the Intermediate Dispersion Spectrograph and Imaging System(ISIS), attached to the William Herschel Telescope WHT. The aim of these observations is to verify the presence of a double-nuclei in its centre, i.e. to confirm whether this object is a dual AGN, with kpc BH separation, or a binary AGN, with a BH separation of a few tens of pc. Or, on the contrary, whether the double-peaked lines are associated with AGN winds/outflows or disk rotation ([10], [22], [7], [17]). The new optical data presented in this work allow us to establish that this object is a triple-peaked narrow emission line object. This is also confirmed after re-modelling previous SDSS-DR7 data obtained 13 years before. Mid-infrared high-resolution images obtained with CanariCam attached to the Gran Telescopio Canarias GTC of Mrk 622, confirm that the nuclear region of Mrk 622 is unresolved at $11.6 \mu \mathrm{m}$. Since Mrk 622 has been previously found to be a double-peaked emission line object, the profile fitting was first done assuming two Gaussian components. The spectral analysis was done using IRAF/specfit 21 .

\footnotetext{
${ }^{1}$ The NASA/IPAC Extragalactic database (NED) is operated by the Jet Propulsion Laboratory, California Institute of Technology, under contract with the National Aeronautics and Space Administration.
} 

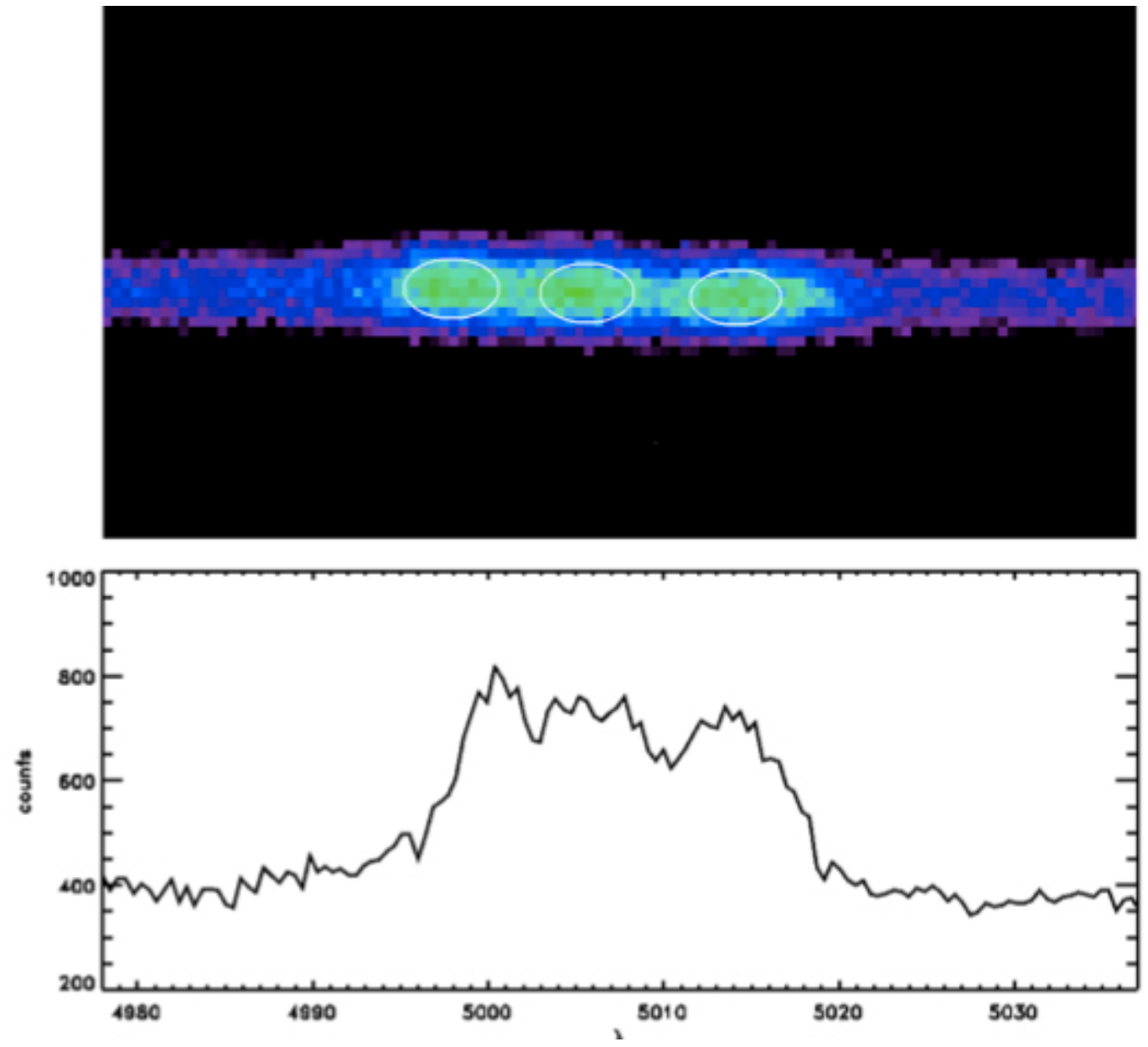

Figure 1: 2D spectrum of the [OIII] $5007 \AA$ region. the three peaks can be noticed

The [OIII]4959,5007 ̊ lines were first fitted. The profile fitting was done assuming the same Full Width at Half Maximum (FWHM) for the two Gaussian components fitted to the [OIII]4959,5007 lines, and for the rest of the lines in the red and blue spectral regions. Modelling the blue and the red part with two Gaussian components initially provided a good fit for the $\mathrm{H}_{\alpha}$ line, except for two caveats. First, it was necessary to include an additional $\mathrm{H}_{\alpha}$ broad component with a FWHM $\sim 1700$ $\mathrm{km} \mathrm{s}^{-1}$ to obtain a good fit. This result contradicts previous classifications of the object as a Sy2 galaxy. On the other hand, we found inconsistencies in the $\Delta \mathrm{v}$ velocity offsets between the blueand red-shifted components, varying from 0 to $470 \mathrm{~km} \mathrm{~s}^{-1}$. To verify this preliminary result, the 2dimensional (2-D) spectra obtained with the WHT were revisited. This analysis showed that there are, in fact, three clearly spatially separated emitting regions in the [OIII]4959,5007 $\AA$ emission line complex (see Figure 1). These regions can be seen in each of the two spectra obtained before, combining them. Between the blue- and red-shifted components the angular projected spatial offset is $0.81 \pm 0.4$ pixels as estimated using IRAF/IMCENTROID. This corresponds to 0.16 ", or $76 \mathrm{pc}$ at the distance of Mrk 622. Based on this result, a new model is proposed to fit these three [OIII] components using three Gaussian profiles. To be consistent, the same model was applied to $\mathrm{H}_{\alpha}$, and to the rest of the emission lines usually studied in the red spectral region. The results obtained 
assuming three Gaussian components are shown in Figure 2.

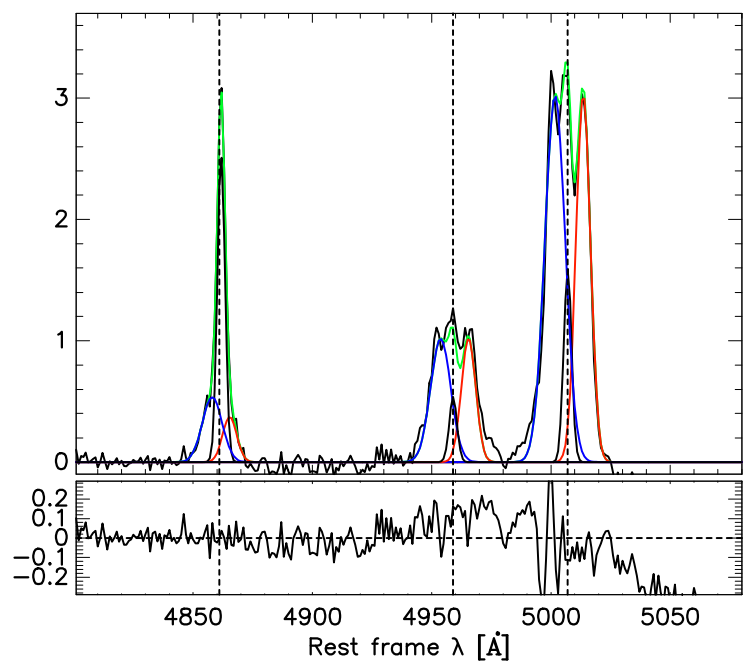

Figure 2: Fits of the [OIII] wavelength region. Fits of the two Oxygens plus $\mathrm{H}_{\beta}$ are shown. The colour code is for all three lines red blue and green for the red component, blue component, and central component respectively. Bottom panel: residuals

It is interesting to note that with the three Gaussian components model, the central Gaussian component is always found to be at the systemic velocity of the galaxy. This central Gaussian component resulted also to be the narrowest of the three. The other two appear to be much broader, and coincide with the blue- and red-shifted components. These components are shown in blue and red colours in Figure 2. Based on the results discussed above, the three Gaussian components model is the best model for fitting the three peaks in Mrk 622 and also provides consistent values of the the velocity offsets for all the emission lines. In order to double check our results, a similar analysis was done to the SSDSS-DR722 public spectrum of Mrk 622. The SDSS rest-frame pure AGN spectrum was fitted following the same procedures used with the WHT data, i.e. the best fit was obtained when 3-Gaussian components were used to fit all the emission lines. It is worth noticing that we get similar results with this spectrum. Indeed, we have a central component that is the narrowest of the three, similar to what we obtained with the WHT spectrum. In addition, blue and red shifted Gaussians were used to obtain the best fit. Note that the WHT spectra were taken 13 years after the SDSS -DR7 spectrum.

\section{Discussion}

The usual way to separate the $\mathrm{SB}$ regions from AGN and low luminosity AGN is done using the BPT empirical diagnostic diagrams. These diagrams use the optical ratios $[\mathrm{OIII}] / \mathrm{H}_{\beta}$, and $[\mathrm{NII}] / \mathrm{H}_{\alpha}$. To obtain an optical classification for Mrk 622 using the data analysed in this work, the BPT diagrams was built. We used the profile analysis done to the WHT data. The locus of the intensity ratios obtained in the diagrams show that the blue- and red-shifted narrow components appear in the AGN region, whereas the central narrow line component appears in one diagram in the compositeHII region, see Figure 3. 


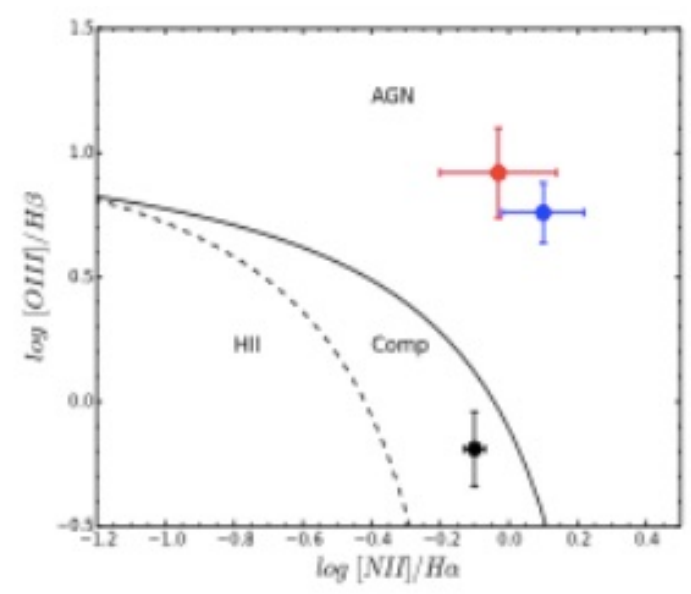

Figure 3: The three peaks in a BPT diagram. Note how the blue and red-shifted components lay in the AGN region, while the systemic velocity region lays in the Starburst/HII region

These results can be interpreted with three different scenarios: 1) A binary AGN with a spatial separation of only $76 \mathrm{pc}$. The presence of two nuclei is not resolved with the MIR data since CanariCam is unable to resolve regions smaller than $0.3 \mathrm{arcsec}$, which at the distance of Mrk 622, $\mathrm{D}=101.9 \mathrm{Mpc}$, is $175 \mathrm{pc}$. Neither the current archival HST observations do resolve the two nuclei. Further information on the CanariCam data is given in a companion paper ([3]). 2) An ionized wind or outflow produced by the AGN responsible for the blue- and red-shifted components with a velocity offset of $500 \mathrm{~km} / \mathrm{s}$. 3) An extended narrow emission line region participating of the rotation of a disk. Scenarios 2 and 3 are ruled out because a central AGN with either a strong ionised wind or a rotating disk cannot plausibly accommodate a SB region in their midst. Tough see $[11,15]$ who find a triple peak in SS433. SS433 contains a binary star where each of the stars have their jets. Nearby there is also a molecular cloud called W50, where there is strong star formation. Although the case could be similar there are a number of differences with Mrk 622. The most important one is that the spectrum of SS433 changes from day to day, showing triple peaks one day, single peak the next day, or even double peaks. The precession of the jets is easily seen in the spectrum pattern. Moreover, the W50 starburst is quite far from the binary stars. [4] claims that the distance from W50 to the SS433 binary stars is $3 \mathrm{kpc}$. In our case, the triple lines have been steady for at least 13 years, that is the time lapse between the Sloan spectrum and our WHT spectrum. Contrary to SS433, our data clearly show a SB region between the two AGN. Having a SB region between two close AGN may seem strange at first sight. Nevertheless, there is a similar example in the Antennae, where two nuclei are in a collision process, and in the way they are forming large amounts of stars ([24]). The difference with Mrk 622 is only that the two nuclei in the Antennae are much further away. This suggests that the Mrk622 nuclei being closer is at a later merging stage. So indeed it seems that there is an SB region between the two nuclei in Mrk622, and this is not the only example. How could that occur? A plausible explanation is, as a consequence of the merger of two galaxies, both AGN. In the case of Mrk 622 the SB originated in the inter-nuclei region. Recently, ([19]) showed how to form stars in a hostile environment. Indeed they showed how massive star-clusters with large SFR evolve in the positive star formation feedback condition. 
In this scenario radiative cooling would reduce the gas temperature, which in turn would produce a stronger cooling and recombination, leading fatally to its collapse and to the formation of new stars. In summary, this mechanism allows producing a SB in compact regions, where winds from previous episodes of star formation coalesce and cool down, retaining the material for further star formation. In our case we are also in a hostile environment, amidst two AGN. This scenario could produce in the inter-nuclei region an area where the conditions explained above hold. Indeed, it is possible that the winds coming from the two nuclei collide and cool down thus facilitating the formation of new stars. This supports the idea that we have two nuclei, and that the ionised AGN wind/outflow of each one collides and thermalises, producing a SB event in the inter-nuclei region.

\section{Conclusions}

We have confirmed an unresolved binary AGN using spectroscopy. This reminds us of the discovery of spectroscopic variable stars. In that case, all we knew was that there were an unresolved source with a velocity shift. In our case, our new data tell us that Mrk 622 presents triple peaked narrow emission lines. This has also been confirmed with the SDSS-DR7 spectra taken 13 years before. This result have lead us to an interpretation with the blue- and red-shifted emission lines coming from two AGN orbiting around each other, plus a central emission line, narrower that the formers, corresponding to a central SB region. The SB region in Mrk 622 is the key element that rules out possible scenarios such as rotating disks, outflows or winds, thus paving the way towards the binary AGN interpretation as the most plausible scenario.

\section{References}

[1] Begelman, M. C., Blandford, R. D. \& Rees, M. J. 1980, Nature, 287, 307-309.

[2] Benítez, E. et al. 2013, Ap. J. 763, L56.

[3] Benítez, E. et al. 2017, MNRAS 474, 36.

[4] G. M. Dubne, et al. 1998, A. J., 116, 1842-1855.

[5] Comerford, J. M. et al. 2009, ApJ. Lett 702, L82-L86.

[6] Comerford, J. M., Schluns, K., Greene, J. E. \& Cool, R. J. 2013, Ap, J. 777, 64.

[7] Crenshaw, D. M., Schmitt, H. R., Kraemer, S. B., Mushotzky, R. F. \& Dunn, J. P. 2010, Ap. j. 708, 419-426.

[8] Gabányi, K. E. et al. 2016, Ap. J. 826, 106

[9] Ge, J.-Q., Hu, C., Wang, J.-M., Bai, J.-M. \& Zhang, S. 2012, Ap J. Suppl. 201, 31.

[10] Heckman, T. M., Miley, G. K. \& Green, R. F. 1984, Ap. J. 281, 525-534.

[11] R. M. Hjellming, K. J. Johnston, Ap. J. 246, L141.

[12] Kauffmann, G. et al. 2003, MNRAS, 346, 1055-1077.

[13] Kewley, L. J., Dopita, M. A., Sutherland, R. S., Heisler, C. A. \& Trevena, J. 2001, Ap. J. 556, $122-140$.

[14] Kewley, L. J., Groves, B., Kauffmann, G. \& Heckman, T. 2006, MNRAS 372, 961-976.

[15] B. Margon, \& S.F. Anderson, 1989, Ap. J., 347, 448-454. 
[16] Milosavljevic, M. \& Merritt, D., 2001, Ap. J. 563, 34-62

[17] Muller-Sánchez et. el. 2015, Ap. J. 813, 103.

[18] Shuder, J. M. \& Osterbrock, D. E. 1981, Ap. J. 250, 55-65

[19] Silich, S. \& Tenorio-Tagle, G., 2013 Ap. J. 765, 43

[20] Volonteri, M., Haardt, F. \& Madau, P. 2003, Ap. J. 582, 559-573

[21] Wang, J.-M. et al. 2009, Ap. J. Lett. 705, L76-L80.

[22] Xu, D. \& Komossa, S. 2009, Ap. J. Lett. 705, L20-L24.

[23] Yu, Q. 2002, MNRAS 331, 935-958

[24] Renaud, F., Bournaud, F., Kraljic, K. \& Duc, P.-A. 2014, MNRAS 442, L33-L37.

[25] Shangguan, J. et al. 2016, Ap. J. 823, 50.

[26] Walter, F. , Decarli, R., Carilli, C. et al. 2012, Nature, 486, 233 .... 